\title{
Diagnostics and treatment of ocular complications in infantile nephropathic cystinosis
}

\author{
Małgorzata Kowalczyk', Mario Damiano Toro ${ }^{1,2}$, Robert Rejdak $\oplus^{1}$, Przemyslaw Sikora ${ }^{3}$ \\ ${ }^{1}$ Department of General Ophthalmology and Paediatric Ophthalmology Service, Medical University of Lublin, Lublin, Poland \\ ${ }^{2}$ Faculty of Medicine, Collegium Medicum Cardinal Stefan Wyszynski University, Warsaw, Poland \\ ${ }^{3}$ Department of Paediatric Nephrology, Medical University of Lublin, Lublin, Poland
}

\begin{abstract}
Cystinosis, as a rare disease, faces many difficulties with regard to appropriate early diagnostics and treatment. The aim of our study is to present current methods of diagnostics and treatment of ocular complications in an infantile nephropathic type of cystinosis. It is the most severe type, causing many ocular and life-threatening systemic complications. Ocular severe complications are usually due to the presence of an infantile nephropathic form of cystinosis, long-term illness, delayed diagnosis, non-compliance, or inappropriate treatment. Slit-lamp biomicroscopy is a major examination detecting corneal cystine crystals and the main tool in cystinosis diagnostics. Anterior segment optical coherence tomography (AS-OCT) and in vivo confocal microscopy (IVCM) are other helpful devices in monitoring the accumulation of crystals in the cornea.
\end{abstract}

KEY WORDS: cystinosis; infantile nephropathic cystinosis; ocular cystinosis

Ophthalmol J 2021; Vol. 6, 206-214

\section{INTRODUCTION}

Cystinosis is one of 6,000 rare diseases in $\mathrm{Eu}-$ rope, according to the European Organisation for Rare Diseases (EURORDIS). It is an autosomal recessive lysosomal storage disease with an incidence of approximately 1 case per 100.000-200.000 live births [1]. The first description of cystinosis was published in 1903 by the Swiss biochemist and physiologist Emil Abderhalden (1877-1950) [2]. The disorder results from mutations in the gene CTNS [3] mapped on chromosome 17p13.2, encoding the protein cystinosin, the lysosomal membrane cystine transporter [4]. The mutations cause defective transport of cystine out of the lysosomes, leading to its excessive accumulation and crystallization in numerous tissues, including initially the kidneys and eyes, and later thyroid, testes, pancreas, muscles, brain, bone marrow, liver, and spleen, causing various clinical symptoms $[1,3]$. Although cystinosis is a monogenic disease, there are three different types, which differ in clinical presentation and severity of the mutation. The most frequent and severe phenotype ( $95 \%$ of cases) is the infantile nephropathic form (MIM:219800, ORPHA411629), the juvenile nephropathic form is less common (MIM:219900, ORPHA411634), and the last one, 


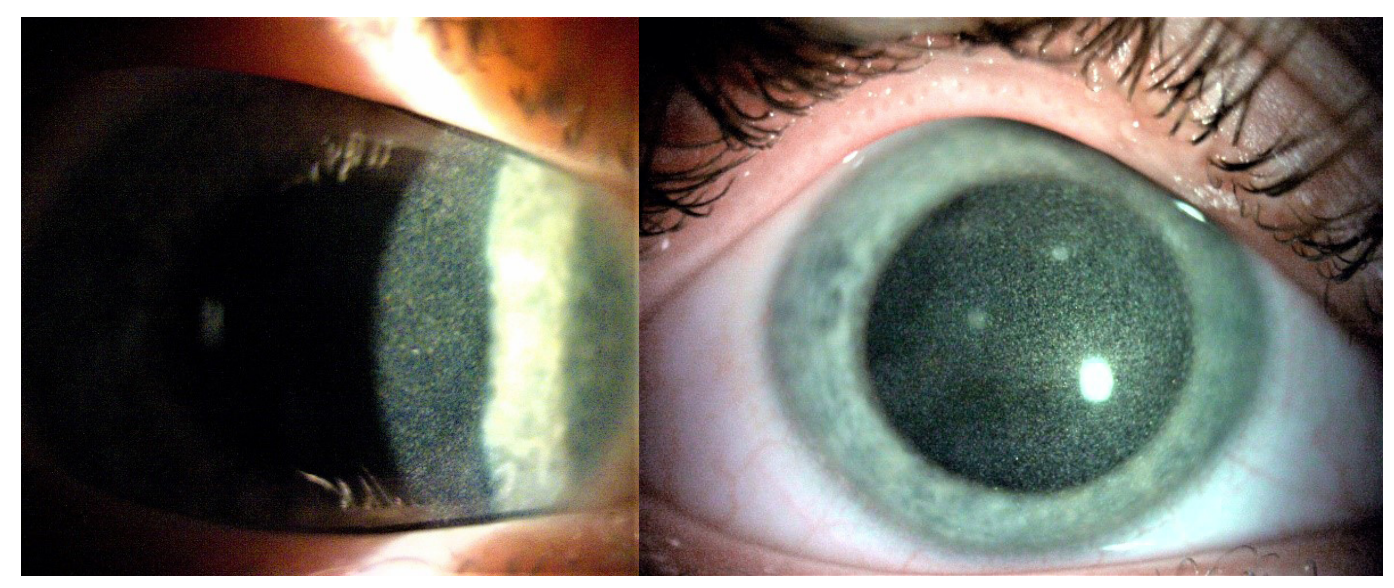

FIGURE 1. Slit-lamp images of crystals deposition in the cornea visible in a narrow and wide slit beam

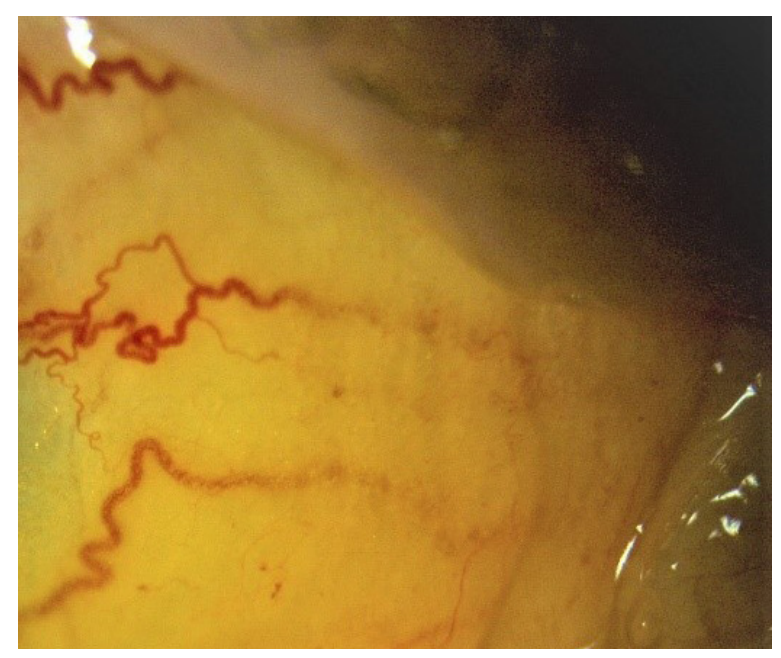

FIGURE 2. Slit-lamp photo displays cystine crystals in the conjunctiva.

extremely rare, is the ocular non-nephropathic form (MIM:219750, ORPHA411641) [5].

\section{OCULAR SYMPTOMS IN CYSTINOSIS}

As we know, renal symptoms are the most prominent features of the nephropathic form of cystinosis. The eyes are the second early affected organ, and corneal crystal formation remains a major ocular complication. Since corneal cystine accumulation is observed in all three types of cystinosis, it is said that the eye is the window to the entire disease, reflecting severity and revealing physiology through the cornea. Although corneal crystal deposits are the main ocular findings, they accumulate in other ocular tissues, leading to mild to severe complications (Fig. 1). Cystine deposits

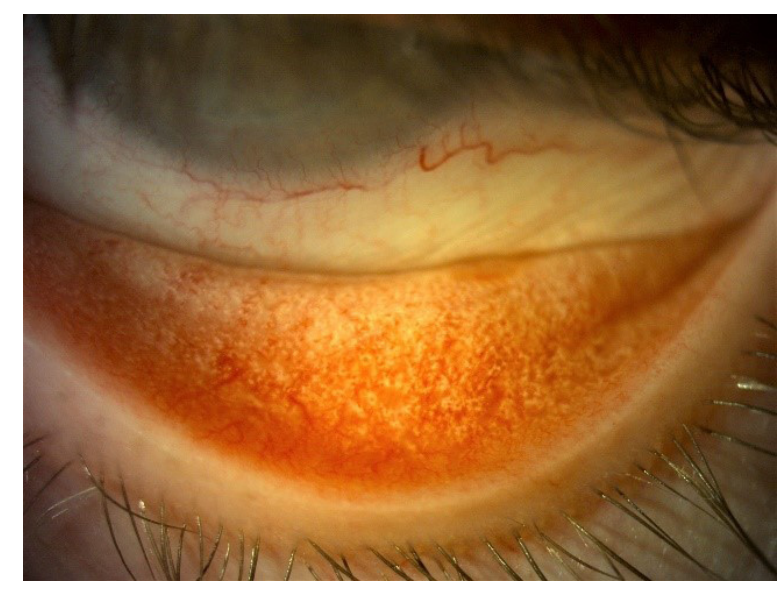

FIGURE 3. Slit-lamp photo presents cystine crystals in palpebral conjunctiva

are seen in the conjunctiva (Fig. 2, 3), iris, lens capsule, ciliary body, choroid, sclera, episclera, extraocular muscles, retinal pigment epithelium, and optic nerve sheath [6]. Corneal crystals are not detectable at birth - they can appear as early as five weeks of age and usually become clinically apparent after 12-16 months [3, 7]. They increase linearly with age and plateau in early adolescence [8]. The crystals are fine, needle-shaped, and highly reflective, diffusely distributed within the cornea. Initially, they appear in the anterior periphery of the cornea and progress centrally and posteriorly [9]. They accumulate progressively with age but at different rates in different patients, rarely affecting central vision. The most ocular complications involving the cornea include: superficial punctate keratopathy, recurrent erosions, filamentary keratitis, calcific band keratopathy (Fig. 4, 5), peripheral neovascularization (Fig. 4-6), superficial limbal cal- 


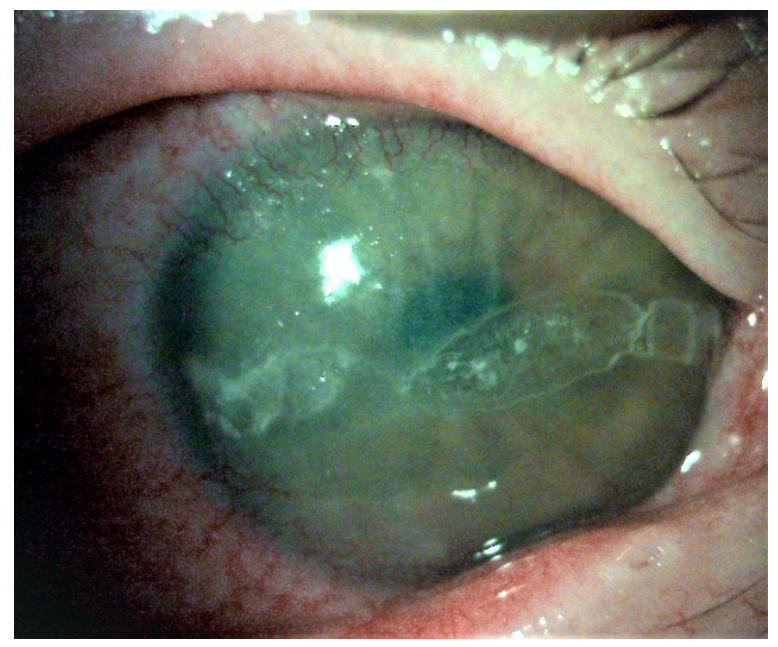

FIGURE 4. Slit-lamp image displays intensified peripheral neovascularization of cornea and band keratopathy in an affected patient with long-term and late initiated treatment of cystinosis

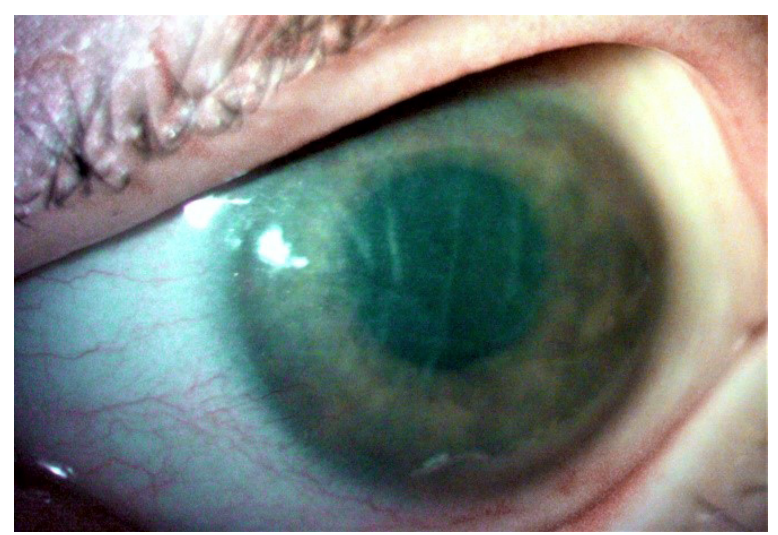

FIGURE 5. Slit-lamp image presents ocular surface complications in the form of cystine crystals in the cornea, hyperaemia of the conjunctiva, peripheral cornea neovascularization, band keratopathy, and Descemet membrane folding

cification, limbal stem cell deficiency (Fig. 5), corneal neurodegeneration and ulcerative keratitis [5]. Sometimes we note conjunctivitis with different grade of hyperemia (Fig. 7). A less frequent complication is glaucoma caused by crystal deposition in the trabecular meshwork or angle-closure with pupillary block due to posterior synechiae $[1,10]$. However, over time patients manifest increasing visual field loss and moderate to severe constriction of the visual field [11]. Chronic uveitis with posterior synechiae associated with iris thickening is also a very rare complication. Hyper- or hypopigmentary retinopathy, macular edema, photoreceptor degeneration, and papilloedema sometimes mani-

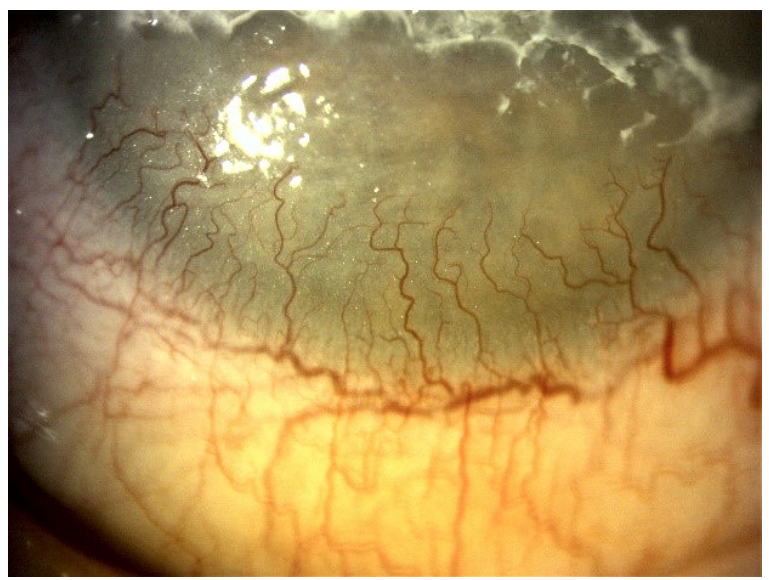

FIGURE 6. Slit-lamp image shows significantly intensified peripheral neovascularization in the cornea in a high magnification slit beam

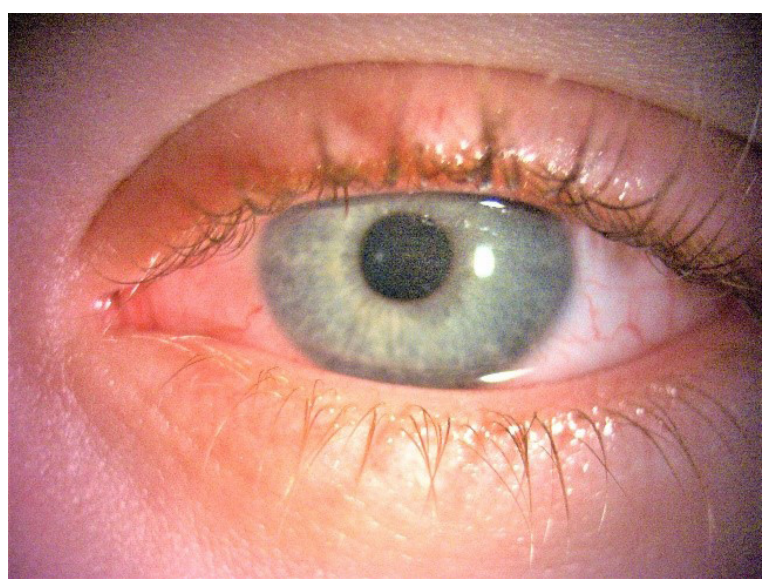

FIGURE 7. Anterior segment a slit-lamp photograph shows cornea cystine crystals and hyperaemia of the conjunctiva

fest in fundoscopic evaluation [12, 13]. Retinal pigment epithelial mottling is the most common posterior segment manifestation and can be seen in infancy [11].

The first and the most frequently reported ocular symptom is photophobia (glare sensitivity), followed by stinging, itching, epiphora, blepharospasm, foreign body sensation, and less often decreased contrast sensitivity [14]. Ocular symptoms usually reflect the extent of corneal crystal accumulation, and they are less severe as the crystals begin to decrease with treatment.

Initially, until the second decade of life, crystal accumulation does not disturb visual acuity, even if the patient is not treated. Severe complications and visual impairment usually start later. 


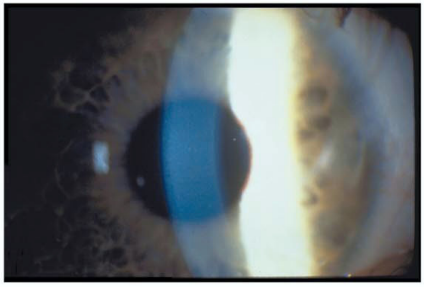

$\operatorname{CCCS}=0.00$

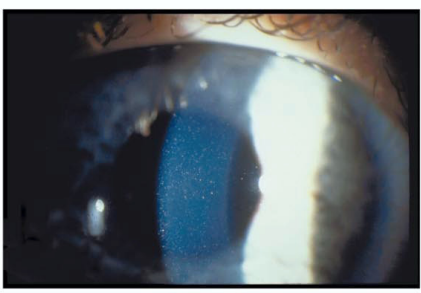

$\mathrm{CCCS}=0.75$

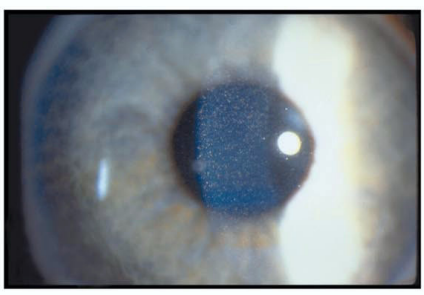

$\mathrm{CCCS}=1.50$

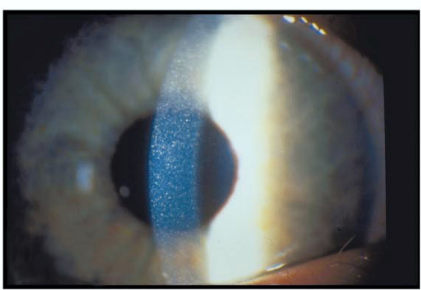

$\operatorname{CCCS}=2.25$

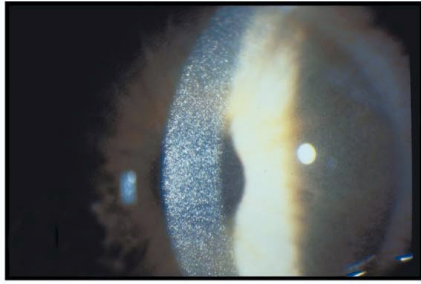

$\mathrm{CCCS}=3.00$

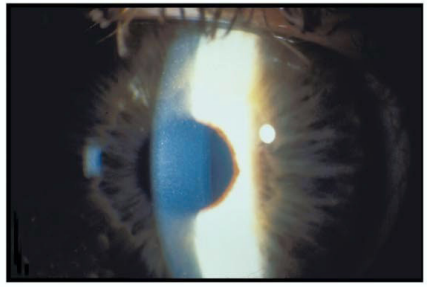

$\operatorname{CCCS}=0.25$

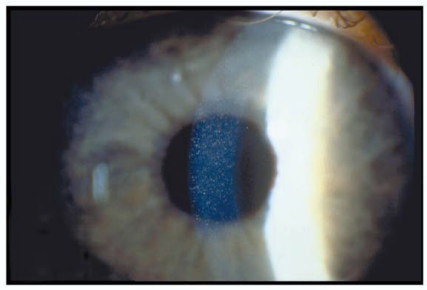

$\mathrm{CCCS}=1.00$

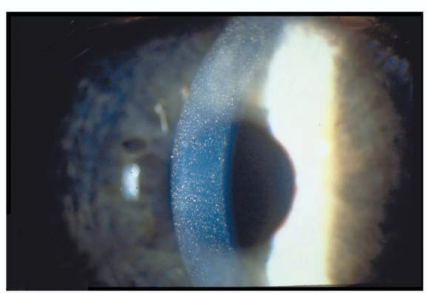

$\mathrm{CCCS}=1.75$

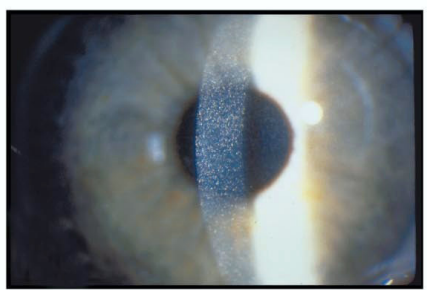

$\mathrm{CCCS}=2.50$

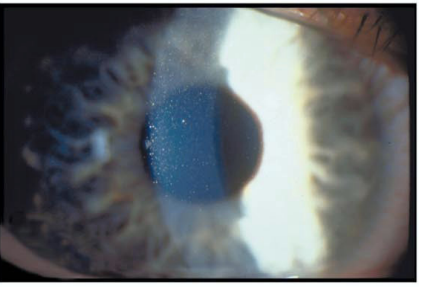

$\mathrm{CCCS}=0.50$

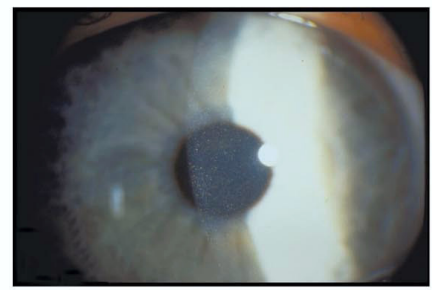

$\mathrm{CCCS}=1.25$

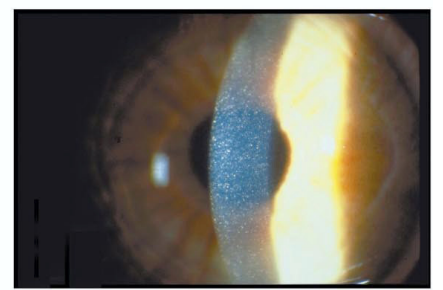

$\mathrm{CCCS}=2.00$

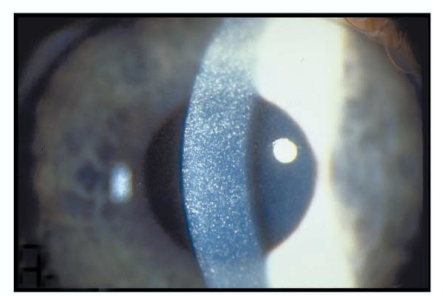

$\mathrm{CCCS}=2.75$

FIGURE 8. Library of slit-lamp photographs showing examples of corneas with corneal cystine crystal scores (CCCSs) of 0 to 3.00 , in increments of 0.25

\section{DIAGNOSIS AND MONITORING OF OCULAR SYMPTOMS}

Accumulated cystine crystals in the anterior segment are detected and assessed by slit-lamp biomicroscopy. It is the simplest and fastest examination, especially when performed by an experienced ophthalmologist.
The semi-quantitative classification created by Gahl et al. allows evaluating the number of accumulated cystine deposits in the cornea using the slit-lamp exam (Fig. 8). It is based on a library of anterior segment photographs and defined as a corneal cystine crystals (CCC) score from $0-3$ in 0.25 unit steps - with increasing crystal densi- 


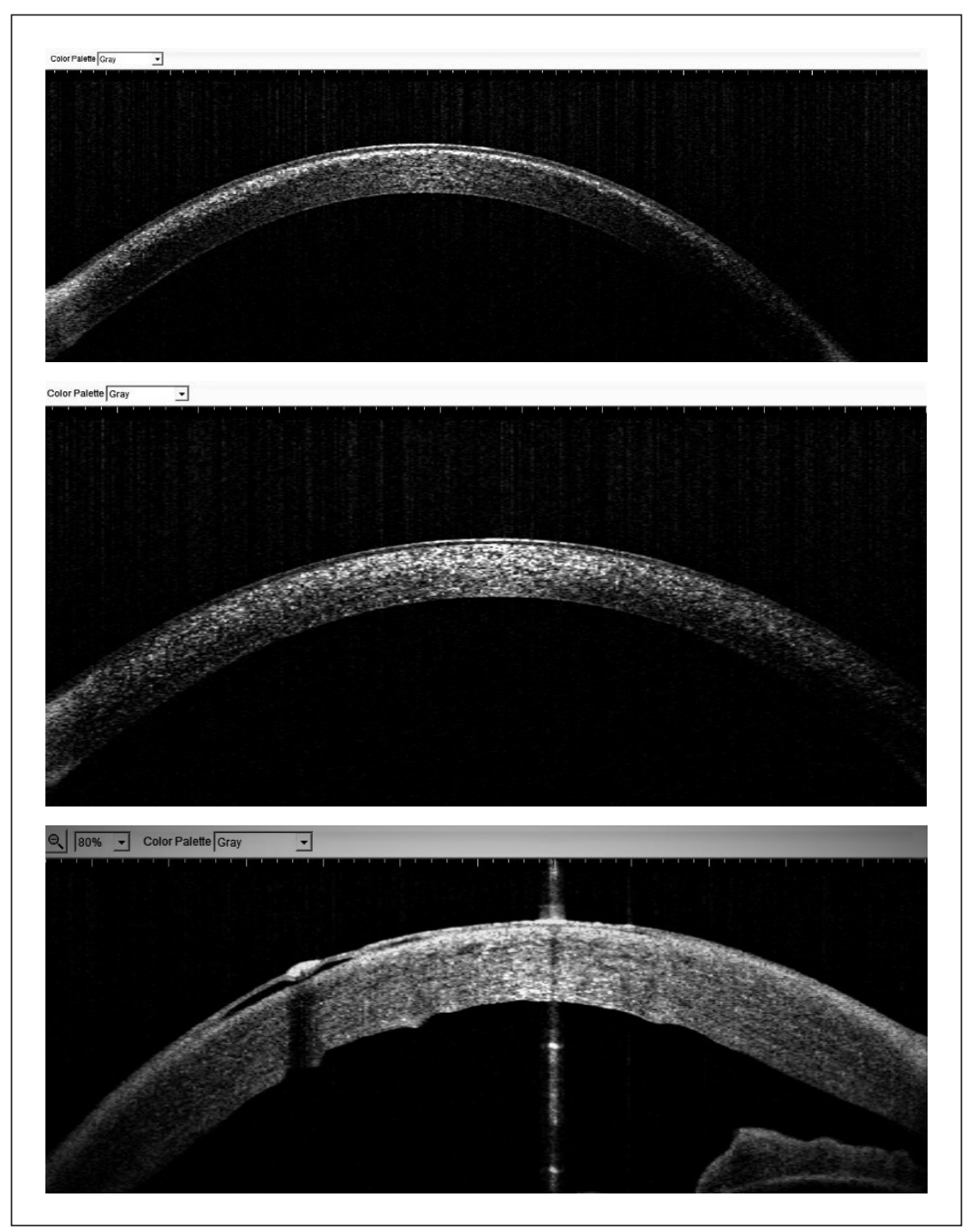

FIGURE 9. Anterior segment optical coherence tomography (AS-OCT) images display the different amounts of cystine crystals in three patients in the cornea. On the top, crystal deposits are visible just in the anterior stroma and the middle — anterior and posterior stroma. The picture below shows thickened cornea with band keratopathy

ties [3]. Anterior segment optical coherence tomography (AS-OCT) allows estimating the depth of crystal deposition $(\mu \mathrm{m})$ in the central cornea using the measurement calipers provided by the AS-OCT software. Due to the non-invasive nature of AS-OCT, the exam can be performed easily, even in very young patients. AS-OCT uses an infrared light source, which is well tolerated and not uncomfortable for patients, even with photophobia. The depth of crystal deposition is expressed as a percentage of the corneal thickness (0-100\%). It is seen as hyperreflective areas beneath the epithelium, taking up different depths of the cornea (Fig. 9). Unfortunately, this imaging method does not allow the exact determination of epithelial and endothelial involvement because of its lower resolu- tion. Corneal pachymetry mapping software can be used to measure corneal thickness [15]. It varies either between patients or between the eyes of the same patient and increases with the number of corneal deposits. AS-OCT valuation of corneal thickness is a highly reliable method. Corneal scarring as one of the complications of cystinosis makes exact measurements of crystal accumulation impossible because they are not visible in the scar area.

In vivo confocal microscopy (IVCM) provides very high-resolution images of corneal layers due to its higher magnification (approximately 800x) and higher resolution $(1-2 \mu \mathrm{m} / \mathrm{pixel})$. It is more advantageous than AS-OCT for assessing crystals deposits because it enables detailed investigation of the ocular surface at the cellular level [16]. This 


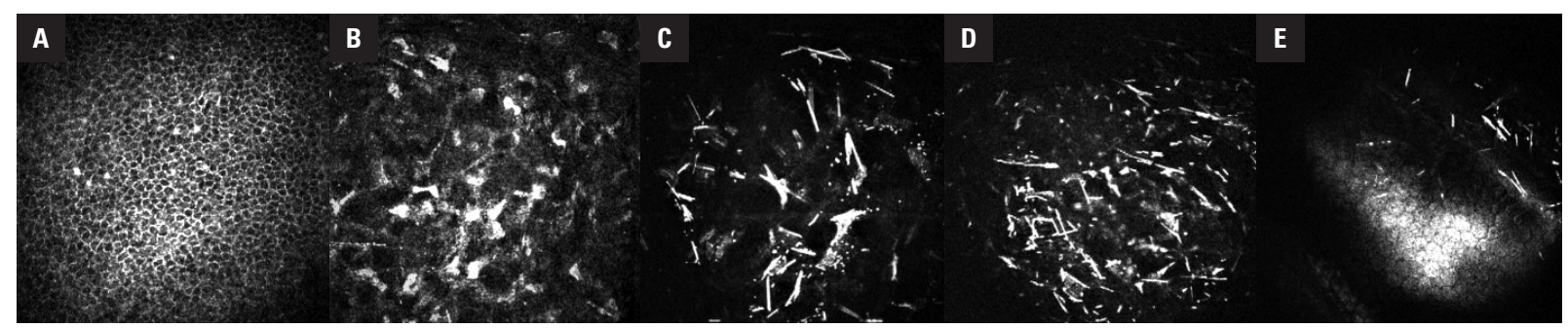

FIGURE 10. In vivo confocal microscope images present accumulation of cystine crystals in each layer of the cornea: A. Epithelium; B. Bowman layer; C. Anterior stroma; D. Deep stroma; E. Endothelium

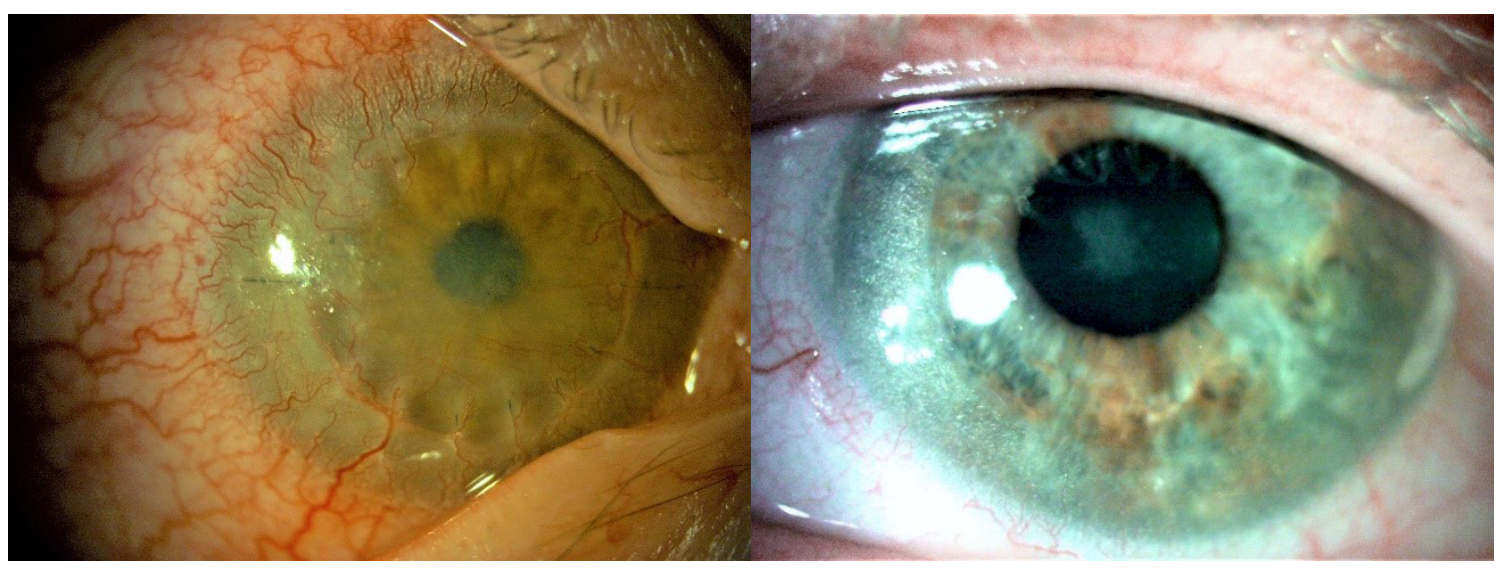

FIGURE 11. Slit-lamp images of post-transplant corneas in two affected patients. The picture on the left shows features of transplant rejection: hazy cornea with increased peripheral neovascularization. On the right, the post-transplant cornea is clear. Crystal deposits are seen only in the host cornea

technique cannot be performed on patients who have difficulties with contact examinations. Especially in children, this exam is a huge challenge. We can assess the number of crystals in each of the five layers of the cornea: the epithelium, Bowman's membrane, anterior stroma, deep stroma, and endothelium (Fig. 10). IVCM makes it possible to quantify the crystal accumulation over a $400 \mathrm{x}$ $400 \mu \mathrm{m}$ area of the central cornea and classify it as 0 if there are no crystals, one if covering $25 \%$, $2-25-50 \%, 3-50-75 \%$, and $4-75 \%$ and more of the defined area. The IVCM score is beneficial in patient follow-up. It remains stable or decreases when there is an appropriate treatment. It also enables the comparative evaluation of crystal density and arrangement in the different layers of the cornea. IVCM technology also allows the visualization of changes in other corneal structures, such as cells, blood vessels, nerves, and connective tissues. It is a good tool for monitoring crystal deposition, inflammatory cell infiltration, and nerve damage [17]. It allows visualising crystals in the cornea and the limbus, and the conjunctiva [15].
IVCM as a contact examination sometimes causes subtle cornea erosions like during other examinations using contact techniques.

Slit-lamp biomicroscopy, AS-OCT, and IVCM examinations are very helpful in monitoring the accumulation of crystals in the anterior segment. Fundoscopy and optical coherence tomography (OCT) are useful in monitoring fundus complications. Fundoscopy is documented by the fundus camera and additionally changes related to the disc (disc volume and retinal nerve fibre layer) and macula using OCT technology. Electroretinography (ERG) detects fundus complications connected with hypo- or hyperpigmentary retinopathy.

Patient complaints, such as photophobia, stinging, and blepharospasm, are evaluated using different scales. Self- and clinician-assessed photophobia can be graded using the Liang et al. cystinosis photophobia scaling system on a scale of 0 to 5 (Tab. 1). For the first time, it was published for different ocular surface inflammations [18]. Self-assessed stinging and blepharospasm are noted on the following scale: none, light, mild and severe. 


\section{TREATMENT}

Oral and topical treatment is required in the management of nephropathic cystinosis. Cysteamine therapy is the only available treatment. It should start as soon as the diagnosis is made, immediately after birth, thanks to which the progression of the disease will significantly slow. As a depleting agent, cysteamine reduces cellular cystine concentration of more than $90 \%$ of the accumulated cystine in all body tissues [7]. Oral and topical treatment slows down disease progression preventing serious systemic and ophthalmic complications. Cystagon ${ }^{\circ}$ (cysteamine bitartrate) distributed by Orphan Europe is the main medication used in the systemic therapy of nephropathic cystinosis. It is critical to take it every six hours orally. The second drug is Procysbi ${ }^{\oplus}$ (cysteamine bitartrate), intended for affected individuals aged one and older. It is not known if it is safe and effective in children under one year of age. Procysbi $i^{\oplus}$ comes in the form of delayed-release capsules and delayed-release oral granules taken every 12 hours.

The most common side effects of systemic cysteamine therapy are nausea and vomiting because of its unpleasant taste and odour (the above data is based on pharmaceutical company information). Cysteamine eye drops dissolve the crystals and alleviate symptoms. To date, there are two kinds of eye drops: hospital preparation eye drops with $0.5 \%$ cysteamine hydrochloride, a licensed preparation of 0.55\% Cystadrops ${ }^{\mathrm{TM}}$ (Orphan Europe, France) distributed in Europe, and $0.44 \%$ Cystaran $^{\mathrm{TM}}$ (Hi-Tech Pharmacal Co., USA) in the USA. Locally prepared $0.5 \%$ cysteamine has been used off-license, and its efficacy, the same as $0.44 \%$ CystaranTM is limited, possibly due to the inconvenience of frequent administration 10-12 times a day. In addition, these are aqueous solutions with no viscosity agent, so the duration of contact with the cornea's surface is insufficient. CystadropsTM as a gel solution with high viscosity is significantly more effective, and its administration is reduced to four times per day [18]. The most frequently reported ocular side reactions are sensitivity to light, redness, blurred vision, and increased lacrimation [19]. It is important that oral administration of cysteamine does not dissolve corneal crystals due to avascular cornea. Ophthalmologic problems are usually treated symptomatically. They appear when cysteamine eye drops are unavailable for the patient or with bad compliance. Photophobia can be relieved by sun avoidance, sunglasses, and lubrication. Some symptoms reported by cystinosis patients, such as itching, stinging and foreign body sensation, can be alleviated using artificial tears. More severe corneal complications often require anti-inflammatory treatment like steroids, antibiotics, or cyclosporine eye drops. Calcific band keratopathy as a complication of long-standing ocular surface inflammation is removed using EDTA chelation. Keratoplasty is rarely required and recommended, especially in cases with intensified corneal neovascularization and limbal stem cell deficiency leading to recurrent ulcerative keratitis. Management of patients after keratoplasty is complicated and challenging. Due to the disease's severe and persistent nature, we note cases with transplant rejection (Fig. 11). There is no symptomatic treatment for retinal involvement in the form of pigmentary retinopathy with photoreceptors degeneration. It is unfortunately irreversible and contributes to vision deterioration.

There are seven patients with cystinosis who regularly have their follow-up visits in our Department: four children aged 9, 12, and 13 with infantile nephropathic form and one child aged 15 with the juvenile nephropathic form; and three adult pa-

\begin{tabular}{|c|c|c|}
\hline Grade & Self-assessed photophobia & Clinician-assessed photophobia \\
\hline 0 & No photophobia & $\begin{array}{l}\text { No photophobia under the slit-lamp beam even with the largest } \\
\text { slit beam }\end{array}$ \\
\hline 1 & Slight difficulty with light causing occasional eye blinking & Photophobia to moderate slit-lamp beam light \\
\hline 2 & Slight difficulty causing regular eye blinking & Photophobia to dimmest slit-lamp beam light \\
\hline 3 & Moderate difficulty with light requiring sunglasses & Inability to tolerate blue light \\
\hline 4 & Severe difficulty requiring almost permanent sunglasses & $\begin{array}{l}\text { Photophobia requiring dark glasses } \\
\text { A patient unable to open eyes inside an illuminated consulting } \\
\text { room }\end{array}$ \\
\hline 5 & $\begin{array}{l}\text { Extreme difficulty with light requiring the patient to stay inside } \\
\text { A patient cannot even tolerate natural light with sunglasses }\end{array}$ & A patient unable to open eyes in a darkened room \\
\hline
\end{tabular}


tients, one aged 24 with the juvenile nephropathic form and two patients aged 34 and 37 with the infantile nephropathic type of cystinosis. The two oldest patients as the only ones who show ocular complications.

\section{DISCUSSION}

Cystinosis is one of the rarest diseases in the world but is found in all ethnic groups with unequal geographical distribution [7]. The first symptoms related to kidneys and eyes appear in the first months of life in patients with the infantile nephropathic form of cystinosis. Cornea cystine crystals are pathognomonic symptoms of the disorder and often help make a diagnosis [9]. Due to the rarity of the disease, in many cases, the diagnosis is often delayed, and proper treatment is initiated too late. This type of cystinosis is usually diagnosed during the first years of life [14]. Systematic ophthalmic follow-up is essential because the accumulation of crystals progresses gradually with age. Anterior segment optical coherence tomography is very helpful in monitoring cystine crystals in the cornea. However, in cases of any ocular surface complications, such as scars, band keratopathy, bullous keratopathy, etc., there is a limitation in evaluating crystal location [20]. We cannot accurately quantify the number of accumulated crystals within the retina using OCT [21]. In vivo confocal microscopy is the gold standard in monitoring cystine accumulation in the cornea [22]. IVCM imaging provides a precise, qualitative evaluation of cornea crystals [15]. IVCM images present cystine crystals and other abnormalities in patients with cystinosis, such as enlarged and abnormal-looking tortuous nerves and beaded nerves in the sub-basal plexus of the cornea [23]. Both AS-OCT and IVCM provide a complete analysis of the cornea and should be used in ophthalmologic follow-up. The medicines used in cystinosis therapy are referred to as "orphan medicines", which means they are used in rare diseases. They can only be obtained with a prescription, and treatment should be started under the supervision of a doctor experienced in the treatment of cystinosis. Ophthalmologic problems are treated with cystine-depleting agents and symptomatically. Cysteamine eye drops dissolving cornea crystals relieve ocular patients' complaints, especially photophobia unless a band keratopathy, pannus, or scars have formed [2]. When corneal cystine accumulation is aggravated, causing limbus stem cell deficiency and peripheral corneal neovascularization, penetrating keratoplasty is performed. $\mathrm{H}$. Liang et al. tried to use anti-VEGF injections in the treatment of corneal neovascularization in cystinosis patients. Unfortunately, the attempt was unsuccessful as, unexpectedly, anti-VEGF injections increased neovascularization. The recurrence of cystine deposition in the corneal allograft is not clear enough. However, we do not note the presence of cystine crystals in the corneal allograft in our patients during eight years of follow-up. Nevertheless, the patients have to be informed about the disease recurrence in the corneal graft because of the persisting underlying disease, and topical cysteamine therapy should be continued. Cystoid macular oedema (CMO) is a severe fundus complication due to the submacular choroidal neovascularization of unknown aetiology in cystinosis patients [9]. Tsilou et al. believe that CMO develops because of vascular endothelial growth factor release following inflammation caused by retinal crystals accumulation. Systemic therapy is critical in reducing cystine concentration in different body tissues, including retinal epithelium, thereby ameliorating or postponing retinopathy [11]. Cysteamine therapy is crucial in treating nephropathic cystinosis patients. If they are left untreated, their life expectancy is up to 12 years [2]. Topical cystine-depleting treatment is also essential in each form of cystinosis.

\section{CONCLUSIONS}

A broad spectrum of ocular pathologic features is related to nephropathic cystinosis. The most serious of them results in a significant visual handicap. Good compliance is essential in preventing severe ophthalmic complications. We hope that the availability of cysteamine therapy will increase each year and make it possible for every affected patient to be treated, thus preventing ocular and systemic complications.

\section{REFERENCES}

1. Pinxten AM, Hua MT, Simpson J, et al. Clinical Practice: A Proposed Standardized Ophthalmological Assessment for Patients with Cystinosis. Ophthalmol Ther. 2017; 6(1): 93-104, doi: 10.1007/s40123017-0089-3, indexed in Pubmed: 28477325.

2. Flockerzi E, Daas L, Schlötzer-Schrehardt U, et al. Ocular changes in nephropathic cystinosis: The course of the gold-dust. Int Ophthalmol. 2019; 39(6): 1413-1418, doi: 10.1007/s10792-018-0954-7, indexed in Pubmed: 29916124.

3. Gahl WA, Kuehl EM, Iwata F, et al. Corneal crystals in nephropathic cystinosis: natural history and treatment with cysteamine eyedrops. Mol Genet Metab. 2000; 71(1-2): 100-120, doi: 10.1006/ mgme.2000.3062, indexed in Pubmed: 11001803. 
4. Elmonem MA, Veys K, Oliveira Arcolino F, et al. Allogeneic HSCT transfers wild-type cystinosin to nonhematological epithelial cells in cystinosis: First human report. Am J Transplant. 2018; 18(11): 2823-2828, doi: 10.1111/ajt.15029, indexed in Pubmed: 30030899.

5. Elmonem MA, Veys KR, Soliman NA, et al. Cystinosis: a review. Orphanet J Rare Dis. 2016; 11: 47, doi: 10.1186/s13023-016-0426-y, indexed in Pubmed: 27102039.

6. Katz B, Melles RB, Schneider JA, et al. Corneal thickness in nephropathic cystinosis. Br J Ophthalmol. 1989; 73(8): 665-668, doi: 10.1136/bjo.73.8.665, indexed in Pubmed: 2765448.

7. Broyer M. Cystinosis. In: Niaudet P. ed. nborn Metabolic Diseases: Diagnosis and Treatment. Springer, Berlin Heidelberg 2012: 617-624.

8. Gahl WA, Kuehl EM, Iwata F, et al. Corneal crystals in nephropathic cystinosis: natural history and treatment with cysteamine eyedrops. Mol Genet Metab. 2000; 71(1-2): 100-120, doi: 10.1006/ mgme.2000.3062, indexed in Pubmed: 11001803.

9. Tsilou E, Zhou M, Gahl W, et al. Ophthalmic manifestations and histopathology of infantile nephropathic cystinosis: report of a case and review of the literature. Surv Ophthalmol. 2007; 52(1): 97-105, doi: 10.1016/j. survophthal.2006.10.006, indexed in Pubmed: 17212992.

10. Sonmez K, Ozcan PY. Angle-closure glaucoma in a patient with the nanophthalmos-ocular cystinosis-foveoschisis-pigmentary retinal dystrophy complex. BMC Ophthalmol. 2012; 12: 23, doi: 10.1186/14712415-12-23, indexed in Pubmed: 22799444.

11. Tsilou ET, Rubin BI, Reed G, et al. Nephropathic Cystinosis. Posterior Segment Manifestations and Effects of Cysteamine Therapy. Ophthalmology, vol. 113, no. 6, pp. 2006; 113(6): 1002-1009, doi: 10.1016/j. ophtha.2005.12.026, indexed in Pubmed: 16603246.

12. Ariceta G, Camacho JA, Fernández-Obispo M, et al. Grupo T-CiS.bcn. Cystinosis in adult and adolescent patients: Recommendations for the comprehensive care of cystinosis. Nefrologia. 2015; 35(3): 304-321, doi: 10.1016/j.nefroe.2015.06.010, indexed in Pubmed: 26523297.

13. Martín-Begué N, Alarcón S, Wolley-Dod C, et al. Intracranial Hypertension in Cystinosis Is a Challenge: Experience in a Children's Hospital. JIMD Rep. 2017; 35: 17-22, doi: 10.1007/8904_2016_18, indexed in Pubmed: 27858370.

14. Biswas S, Gaviria M, Malheiro L, et al. Latest Clinical Approaches in the Ocular Management of Cystinosis: A Review of Current Practice and Opinion from the Ophthalmology Cystinosis Forum. Ophthalmol Ther. 2018; 7(2): 307-322, doi: 10.1007/s40123-018-0146-6, indexed in Pubmed: 30242621.

15. Labbé $A$, Niaudet $P$, Loirat $C$, et al. In vivo confocal microscopy and anterior segment optical coherence tomography analysis of the cornea in nephropathic cystinosis. Ophthalmology. 2009; 116(5): 870-876, doi: 10.1016/j.ophtha.2008.11.021, indexed in Pubmed: 19410944.

16. Zhivov A, Stachs 0, Kraak R, et al. In Vivo Confocal Microscopy of the Ocular Surface. Ocular Surf. 2006; 4(2): 81-93, doi: 10.1016/ s1542-0124(12)70030-7.

17. Liang H, Baudouin C, Tahiri Joutei Hassani R, et al. Photophobia and corneal crystal density in nephropathic cystinosis: an in vivo confocal microscopy and anterior-segment optical coherence tomography study. Invest Ophthalmol Vis Sci. 2015; 56(5): 3218-3225, doi: 10.1167/iovs. 15-16499, indexed in Pubmed: 26024106.

18. Liang H, Labbé A, Le Mouhaër J, et al. A New Viscous Cysteamine Eye Drops Treatment for Ophthalmic Cystinosis: An Open-Label Randomized Comparative Phase III Pivotal Study. Invest Ophthalmol Vis Sci. 2017; 58(4): 2275-2283, doi: 10.1167/iovs.16-21080, indexed in Pubmed: 28426870.

19. Kowalczyk M, Piecyk-Sidor M, Mackiewicz J, et al. Posterior staphyloma in the normal lenght eyeball - a case report. Klin Oczna. 2007; 109(4-6).

20. Kowalczyk M, Toro MD, Rejdak R, et al. Ophthalmic Evaluation of Diagnosed Cases of Eye Cystinosis: A Tertiary Care Center's Experience. Diagnostics (Basel). 2020; 10(11): 911, doi: 10.3390/diagnostics10110911, indexed in Pubmed: 33171856.

21. Al-Hemidan A, Shoughy SS, Kozak I, et al. Efficacy of topical cysteamine in nephropathic cystinosis. Br J Ophthalmol. 2017; 101(9): 1234-1237, doi: 10.1136/bjophthalmol-2016-309278, indexed in Pubmed: 28057644.

22. Veys KRP, Besouw MTP, Pinxten AM, et al. Cystinosis: a new perspective. Acta Clin Belg. 2016; 71(3): 131-137, doi: 10.1179/229533371 4Y.0000000113, indexed in Pubmed: 25560059.

23. Biswas S, Sornalingam K. The Ocular Status of Cystinosis Patients Receiving a Hospital Pharmacy-Made Preparation of Cysteamine Eye Drops: A Case Series. Ophthalmol Ther. 2019; 8(1): 125-136, doi: 10.1007/s40123-018-0156-4, indexed in Pubmed: 30519924. 